\title{
Jupiter Microtubule Associated Homolog 1
}

National Cancer Institute

\section{Source}

National Cancer Institute. Jupiter Microtubule Associated Homolog 1. NCI Thesaurus. Code C114591.

Jupiter microtubule associated homolog 1 (154 aa, $16 \mathrm{kDa}$ ) is encoded by the human JPT 1 gene. This protein plays a role in signal transduction, cell adhesion and cell cycle regulation. 